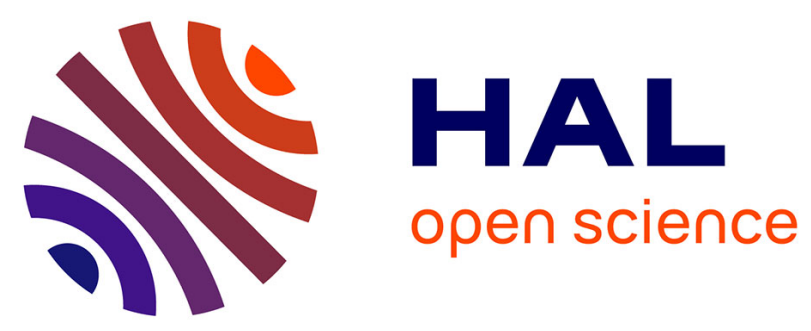

\title{
Spatially asymmetric transients of propagating exciton-polariton modes in a planar CdZnTe/CdMgTe guiding structure
}

J. Vondran, F. Spitzer, M. Bayer, I. Akimov, A. Trautmann, M. Reichelt, C. Meier, N. Weber, T. Meier, R. André, et al.

\section{To cite this version:}

J. Vondran, F. Spitzer, M. Bayer, I. Akimov, A. Trautmann, et al.. Spatially asymmetric transients of propagating exciton-polariton modes in a planar $\mathrm{CdZnTe} / \mathrm{CdMgTe}$ guiding structure. Physical Review B, 2019, 100 (15), pp.155308. 10.1103/PhysRevB.100.155308 . hal-02410215

\section{HAL Id: hal-02410215 \\ https://hal.science/hal-02410215}

Submitted on 3 Dec 2020

HAL is a multi-disciplinary open access archive for the deposit and dissemination of scientific research documents, whether they are published or not. The documents may come from teaching and research institutions in France or abroad, or from public or private research centers.
L'archive ouverte pluridisciplinaire HAL, est destinée au dépôt et à la diffusion de documents scientifiques de niveau recherche, publiés ou non, émanant des établissements d'enseignement et de recherche français ou étrangers, des laboratoires publics ou privés. 


\title{
Spatially asymmetric transients of propagating exciton-polariton modes in a planar CdZnTe/CdMgTe guiding structure
}

\author{
J. Vondran $\odot$, F. Spitzer, M. Bayer, and I. A. Akimov \\ Experimentelle Physik 2, Technische Universität Dortmund, 44221 Dortmund, Germany \\ A. Trautmann, M. Reichelt, C. Meier, N. Weber, and T. Meier \\ Department Physik \& CeOPP, Universität Paderborn, D-33098 Paderborn, Germany \\ R. André and H. Mariette \\ Université Grenoble Alpes, CNRS, Institut NEEL, Grenoble 38000, France
}

(Received 13 May 2019; revised manuscript received 18 September 2019; published 25 October 2019)

\begin{abstract}
We report on ultrafast time-resolved pump-probe studies in a CdZnTe/CdMgTe planar guiding structure covered with a metallic grating. The one-dimensional periodic gold structure allows for efficient coupling into the guiding layer for $p$-polarized $30 \mathrm{fs}$ optical pulses with a large spectral bandwidth of about $60 \mathrm{~nm}$. The resulting spectral width of optical pulses propagating inside the guiding layer corresponds to $20-30 \mathrm{~nm}$. We demonstrate that the excitation of exciton-polariton modes in the guiding layer leads to a modulation of the optical response in the vicinity of the excitonic resonance. Spatially resolved pump-probe measurements show an asymmetric behavior in the optical response when the relative position of the pump and probe spots is varied on the scale of ten micrometers perpendicular to the metal ridges. This is attributed to the excitation of resonant and off-resonant exciton-polariton modes which propagate in opposite directions inside the guiding layer in accordance with their dispersion relations. Two main mechanisms are considered and evaluated, namely, Pauli blocking and excitation-induced dephasing, which are shown to be responsible for the pump-induced changes in the exciton absorption spectrum. While both of these processes lead to the generation of photoexcited carriers in the guiding layer, their impact on the optical properties (transmission and reflection) are different which leads to the asymmetric behavior of the spatially resolved transients.
\end{abstract}

DOI: 10.1103/PhysRevB.100.155308

\section{INTRODUCTION}

Nanophotonic circuits require a precise control over the spatiotemporal propagation of light $[1,2]$. Nonlinear effects can be used as a key feature for light management in novel nanophotonic devices, for example, in delay lines by which the arrival time of ultrashort laser pulses at certain components can be controlled accurately [3]. Also, mixing of optical signals can be used to route the resulting light pulses in the desired direction and time frame. The use of semiconductor nanostructures for these purposes is attractive because their nonlinear optical response is strong at photon energies close to the band gap energy and they can be well implemented into other optical and electronic components with existing technologies. [4] For the design of such systems an accurate knowledge of the dephasing and relaxation of optical excitations and their dependence on the experimental conditions in the linear and nonlinear regimes are required as can be obtained using wave-mixing and pump-probe experiments [5].

Propagation of ultrashort optical pulses at photon energies close to the excitonic resonance in semiconductors deserves special attention. Here, the group velocity for propagation through the crystal may be significantly decreased. This is the result of the efficient coupling between light and excitons leading to the formation of exciton-polaritons (EP) $[6,7]$. Coherent propagation of EPs in thick submillimeter bulk crystals of high quality was studied in GaN (Ref. [8]), $\mathrm{ZnO}$ (Ref. [9]), and CdZnTe (Ref. [10]) structures using time-of-flight measurements. Thereby, propagation pulse delays of light on the order of several hundreds of picoseconds were reported. In CdZnTe bulk crystals, we observed nutation of the EP polarization due to strong enhancement of magneto-optical effects such as the Faraday rotation and the nonreciprocal birefringence [11]. Recently, long-distance EP propagation in bulk CdZnTe crystals was studied using pump-probe techniques, where an off-resonant pump pulse in the transparency region created excess carriers due to two-photon absorption [12].

However, on-chip applications require light management in small and compact volumes. For that reason there is an interest in optical studies of semiconductor-based guiding structures where the EPs are confined in one or two directions. Here, grating structures can be effectively used to tune the light coupling into the semiconductor structures. In particular, dispersion engineering using a metallic [13] and high-indexcontrast subwavelength [14] grating as a mirror in vertical microcavity structures has been performed. Recent studies demonstrated strong light-matter coupling in planar guiding structures $[15,16]$. In addition, new types of nonlinearities, e.g., low-power solitons, emerge due to confinement of the optical field in an InGaAlAs-based wave guiding structure with a quantum well as optically active layer inside [17]. 
Therefore, based on this motivation, we investigate nonlinear optical phenomena in planar wave guiding structures in this work. We focus on CdZnTe structures where large delays for pulse propagation were observed in our previous studies [10-12].

In detail, we investigate pump-probe transients where the short optical pulses are coupled into the guiding layer through a metallic Bragg grating. The pump-induced changes in the optical response are probed at the excitonic resonance. The use of metallic grating couplers at the top of the guiding layer allows efficient excitation with a large spectral bandwidth for $p$-polarized light which is important for operation with ultrashort pulses. Using a spatially resolved technique with spectrally broad, 30-fs optical pulses we demonstrate that different spectral components propagate in different directions leading correspondingly to different impacts on the exciton transition (selective excitation of EPs propagating in different directions). This allows us to distinguish between two main mechanisms that lead to the changes in the optical response. In case of resonant EPs with the photon energy close to the exciton resonance, we observe a bleaching of the absorption. For off-resonant EPs with lower photon energy, the opposite scenario is obtained where the absorption is enhanced due to excitation-induced dephasing. The results demonstrate that these two mechanisms can be used to control the propagation of light in guiding layers.

\section{OPTICAL PROPERTIES OF GUIDING STRUCTURES}

The investigated structure is schematically shown in Fig. 1. The semiconductor part was grown by molecular beam epitaxy on a $\mathrm{Cd}_{0.96} \mathrm{Zn}_{0.04} \mathrm{Te}$ substrate. It is represented by the 700 -nm-thick $\mathrm{Cd}_{0.9} \mathrm{Zn}_{0.1} \mathrm{Te}$ buffer layer followed by the guiding structure. The latter comprises a 170-nm-thick $\mathrm{Cd}_{0.9} \mathrm{Zn}_{0.1}$ Te guiding layer sandwiched between $400-\mathrm{nm}$ and 100 -nm-thick $\mathrm{Cd}_{0.4} \mathrm{Mg}_{0.6} \mathrm{Te}$ cladding layers at the bottom and top, respectively. The composition of $\mathrm{Zn}$ and $\mathrm{Mg}$ in the guiding and cladding layers was selected in order to achieve optimal conditions with relatively large contrast in the refractive indices (about 15\% larger in the guiding layer) in combination with optimal matching of the lattice constant

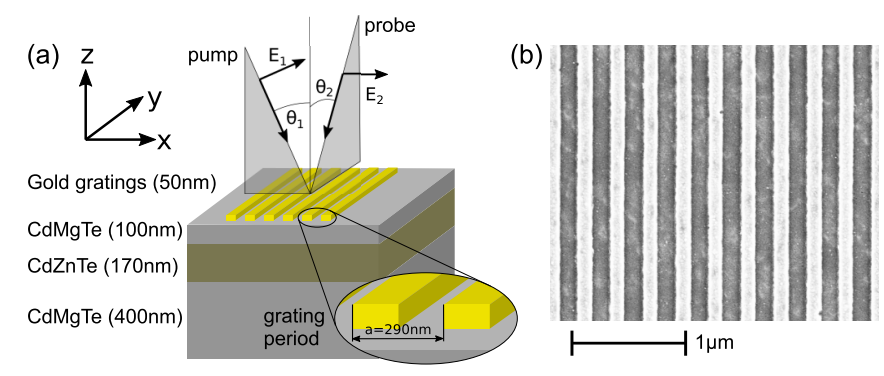

FIG. 1. (a) Scheme of the sample with a gold grating on a CdZnTe guiding film inside of a CdMgTe cladding (layer thickness given in brackets). Also shown is the incidence geometry of the pump-probe experiment: The pump (probe) beam and its electric field $E_{1}\left(E_{2}\right)$ in $p(s)$ polarization as well as the incidence angles $\theta_{1}\left(\theta_{2}\right)$, which corresponds to the polarization configuration in which TM modes are excited. (b) shows a scanning electron microscope picture of the gold grating with period $a=290 \mathrm{~nm}$. in both materials, which ensures low levels of defects and dislocations and excellent optical properties.

In order to couple light into the guiding layer, 50-nm-thick periodically perforated gold gratings were patterned at the top of the semiconductor. Therefore an electron-sensitive resist was deposited on top of the CdMgTe top layer. Then, after exposure with electron beam lithography and development of the resist, only the unexposed areas remain covered. Via electron beam vapor deposition a thin layer $(1 \mathrm{~nm})$ of chrome for improved adhesion and the 50-nm-thick gold layer are placed on top. The final lift-off process of putting the sample in an acetone bath dissolves the remaining resist which removes also the gold strips on the resist. Only the areas (grating strips) which were scanned by the electron beam remain coated with gold. In this work, we study the sample with a period $a=290 \mathrm{~nm}$ and a 1:1 ratio of gold ridge to air gap width. The grating size is $250 \mu \mathrm{m}$ by $250 \mu \mathrm{m}$ which is much larger than the laser spots used in the experiment so that any edge repercussions are excluded from our considerations. The sample area outside the grating remains unpatterned and can be considered as bare guiding structure.

Figure 2 shows the steady state angle-resolved reflectivity spectra in $p$ and $s$ polarizations from the areas patterned with the grating, measured at the temperature $T=10 \mathrm{~K}$. The exciton resonance in the CdZnTe guiding layer is clearly observed at photon energy of about $\hbar \omega_{X} \approx 1.64 \mathrm{eV}\left(\lambda_{X} \approx 755 \mathrm{~nm}\right)$. Its energy as well as strength are almost independent of the polarization and angle of light $\theta$. In contrast to the exciton

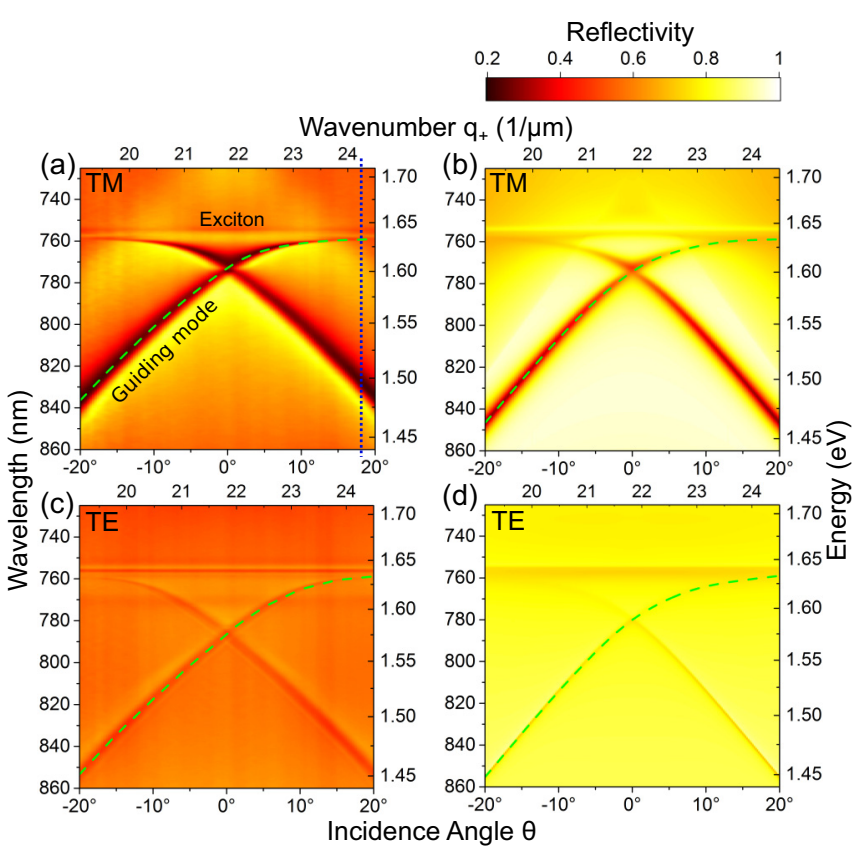

FIG. 2. Steady-state reflectivity spectra from the grating for $p$ and $s$-polarized light $(a=290 \mathrm{~nm})$. (a) and (c) show experimental data at $T=10 \mathrm{~K}$ temperature. (b) and (d) correspond to theory. TM- and TE-waveguiding modes are shown in (a), (b) and (c), (d), respectively. Dashed green lines show the dispersion curves of the guiding modes, i.e., their energies as function of wave number $q_{+}$ (see top horizontal axis and Sec. II for details). The vertical blue dashed line in (a) indicates the excitation by the pump at an angle of $\theta=17^{\circ}$. 


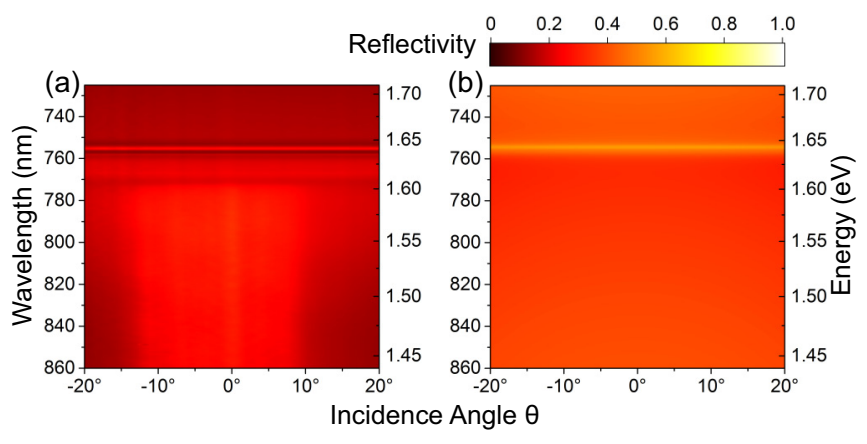

FIG. 3. Steady-state reflectivity spectra from the bare structure without gold grating. Shown are (a) the experimental data measured at $T=10 \mathrm{~K}$ temperature in $p$ polarization and (b) the corresponding results from theory.

resonance, the guiding modes show a strong dependence on polarization and incidence angle. Excitation with linearly $p$ and $s$-polarized light addresses transverse magnetic (TM) and electric (TE) guiding modes, respectively. Here, we observe gradual changes of the resonance position with variation of $\theta$. In addition, the characteristic bending of the dispersion curves at photon energies close to the exciton resonances indicates EP formation accompanied by a strong decrease of the group velocity. The two modes with positive and negative dispersion are present which correspond to excitation of guided electromagnetic waves with wave number $q_{+}=k_{0} \sin \theta+G$ and $q_{-}=-k_{0} \sin \theta-G$, respectively, where $G=2 \pi / a$ is the reciprocal vector of the metallic grating and $k_{0}$ is the wave vector of the incident light. The dispersion curves $\hbar \omega_{g}\left(q_{+}\right)$ for the corresponding modes are shown in Fig. 2 where the frequency of the mode $\omega_{g}$ is defined as the minimum in the reflectivity spectrum. We also measured the reflectivity spectra for structures with grating periods ranging from 230 to $310 \mathrm{~nm}$. In full accord with the expectations the position of the guiding modes shifts to a lower energy with increasing $a$, while the position of the exciton resonance remains unchanged. In Fig. 3, reflectivity spectra from the bare guiding layer without the grating structure are shown. In this case, the exciton feature around $1.64 \mathrm{eV}$ is present while the guiding modes are absent.

From Fig. 2 two distinct differences between TE and TM modes are clearly recognized. First, the positions of the TE modes shift to lower energies with respect to the TM modes for a given angle $\theta$ which is expected for the bare planar waveguide. Second, the TM resonances are much more pronounced. We attribute this observation to additional coupling of the TM guiding modes to plasmonic resonances in the metallic grating which can be excited only with $p$-polarized light [18]. This leads to a significant increase of the TM mode strengths and spectral widths: For instance, at about $820 \mathrm{~nm}$ wavelength for $\theta=17^{\circ}$ incidence angle the guiding mode resonance increases by about four times in strength and three times in spectral width (from $8 \mathrm{~nm}$ to $24 \mathrm{~nm}$ ) going from TE to TM polarization. These findings are confirmed by theoretical calculations performed using the CST MICROWAVE STUDIO software [19], see Figs. 2(b) and 2(d). All simulations are carried out with the frequency domain solver in three dimensions in combination with periodic boundary conditions and Floquet ports. The dielectric properties of the plasmonic gold grating are obtained directly from MICROWAVE STUDIO's material library. The optical properties of the CdMgTe cladding layers are determined using the experimental data from Ref. [20]. In order to describe the excitonic resonance of the CdZnTe guiding layer and the dispersive propagation effects a Lorentz model for the dielectric function $\epsilon(\omega)=$ $\epsilon_{B}+\left(f \omega_{0}^{2}\right) /\left(\omega_{0}^{2}-\omega^{2}+i \omega \Gamma\right)$ is added and the continuum absorption above the exciton is described by a multi-Lorentz approach. The resulting parameters are similar to Ref. [10], namely the exciton resonance frequency $\hbar \omega_{0} \sim 1.642 \mathrm{eV}$, the refractive index $n_{\mathrm{CdZnTe}}=\sqrt{\operatorname{Re}(\epsilon(800 \mathrm{~nm}))} \approx 2.9$, the exciton damping $\hbar \Gamma \sim 1 \mathrm{meV}$, and are adjusted such that they reproduce the experimental results from the reflectivity spectra, Figs. 2(a) and 2(c), well. The large spectral bandwidth of the TM resonances is important for coupling to short optical pulses which spectral width increases with decreasing pulse duration, in our experiments $30 \mathrm{fs}$.

\section{SPATIALLY RESOLVED PUMP-PROBE}

\section{A. Experimental approach}

In what follows we concentrate on time-resolved pumpprobe experiments where the ultrashort optical pulses are coupled through the grating into the guiding layer. For these purposes we use a Ti:Sa laser as light source with $80-\mathrm{MHz}$ repetition rate and a $60 \mathrm{~nm}$ [full width half maximum (FWHM)] wide spectrum centered around $810 \mathrm{~nm}$. The pulse chirp acquired during propagation through the optical elements was compensated by a pulse shaper and a compressor using the multiphoton intrapulse interference phase scan procedure [21]. In that way, the duration of both pump and probe pulses at the sample position are about 30 fs. The pump beam is incident on the sample in the plane perpendicular to the grating ridges while the probe beam is in the plane parallel to the ridges, see Fig. 1. The polarizations of the incident beams are selected to be perpendicular to the grating ridges, which corresponds to $p$ - and $s$-polarized pump and probe beams, respectively. In this case, the most efficient excitation with the pump and sensitive detection with the probe are achieved. Both beams have incidence angles of $\theta_{1}=\theta_{2}=17^{\circ}$ relative to the optical axis ( $z$ axis) with an aperture of about $6^{\circ}$. The laser beams are focused to spot sizes on the sample surface of approximately $12 \mu \mathrm{m}$ in diameter for the pump and $8 \mu \mathrm{m}$ for the probe using different sectors of the same microscope reflective objective [22]. The same objective is also used to collect the reflected probe beam. The energy densities per pulse are $120 \mu \mathrm{J} / \mathrm{cm}^{2}$ (pump) and $40 \mu \mathrm{J} / \mathrm{cm}^{2}$ (probe).

In order to increase the sensitivity, the probe beam reflected from the sample is fed through a bandpass filter with $760 \mathrm{~nm}$ center wavelength, which is close to the exciton resonance wavelength of the sample, and $7 \mathrm{~nm}$ width. The probe beam is detected by one of the diodes of a balanced photodetector while the second diode is exposed to a fraction of the laser beam as a reference to account for the intensity fluctuations of the Ti:Sa laser. The transients are obtained by modulating the pump intensity with a mechanical chopper wheel and demodulating the differential photodetector signal by means of a lock-in amplifier. Finally we measure the differential reflectivity $\Delta R / R$, which is the reflectivity change $\Delta R$ induced 

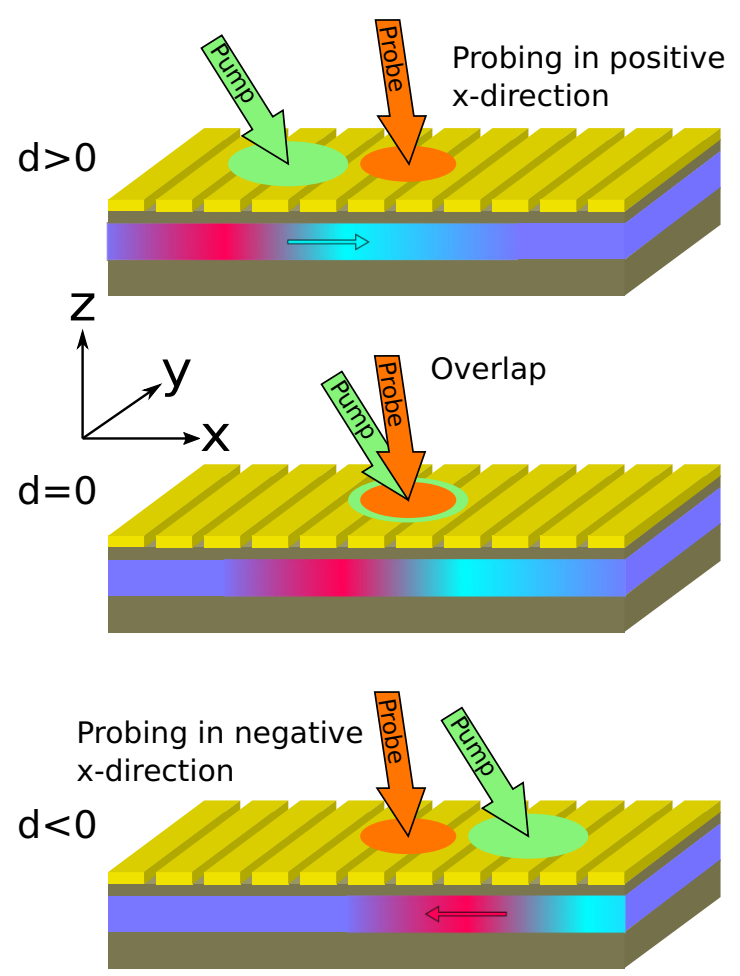

FIG. 4. Schematic presentation of spatial positioning of the pump and probe spots relative to each other ("mapping") on the grating surface. The violet layer (guiding film) illustrates the directional propagation: Red denotes the off-resonant lower energy EP and blue the high energy EP in resonance with the exciton.

by the pump pulse normalized to the overall reflectivity $R$ while the delay time $\tau$ between the pump and probe pulses on the sample is varied by changing the position of a mirrorreflector located on a mechanical delay line. As in the steady state experiments, the sample is held in a helium flow cryostat at a temperature of about $10 \mathrm{~K}$.

Our approach is based on exploring the propagation of the pump pulse in the guiding structure. Therefore we perform spatially resolved pump-probe measurements where the distance between the pump and probe beams at the sample surface can be varied in a systematic and reproducible way. This is accomplished through a mirror which is placed on rotatable piezoelectric positioners to change the pump beam's angle of incidence in the microscope objective, thus changing its spot position on the surface. This allows us to perform spatially resolved "mapping" measurements with a resolution of $4 \mu \mathrm{m}$. With the probe spot kept at a constant position, the pump spot can be moved along the $x$ and $y$ directions, consequently altering its position and the influence of the coupled-in light relative to the spot where the probe measurement is taken. This is shown schematically in Fig. 4: The top part shows the situation where the pump is shifted in negative $x$ direction, while in the bottom part it is shifted in positive $x$ direction. The middle part shows spatial overlap of both spots.

\section{B. Mapping the exciton-polaritons}

Figure 5 shows the differential reflectivity transients for different displacements $d$ between the pump and probe beams.
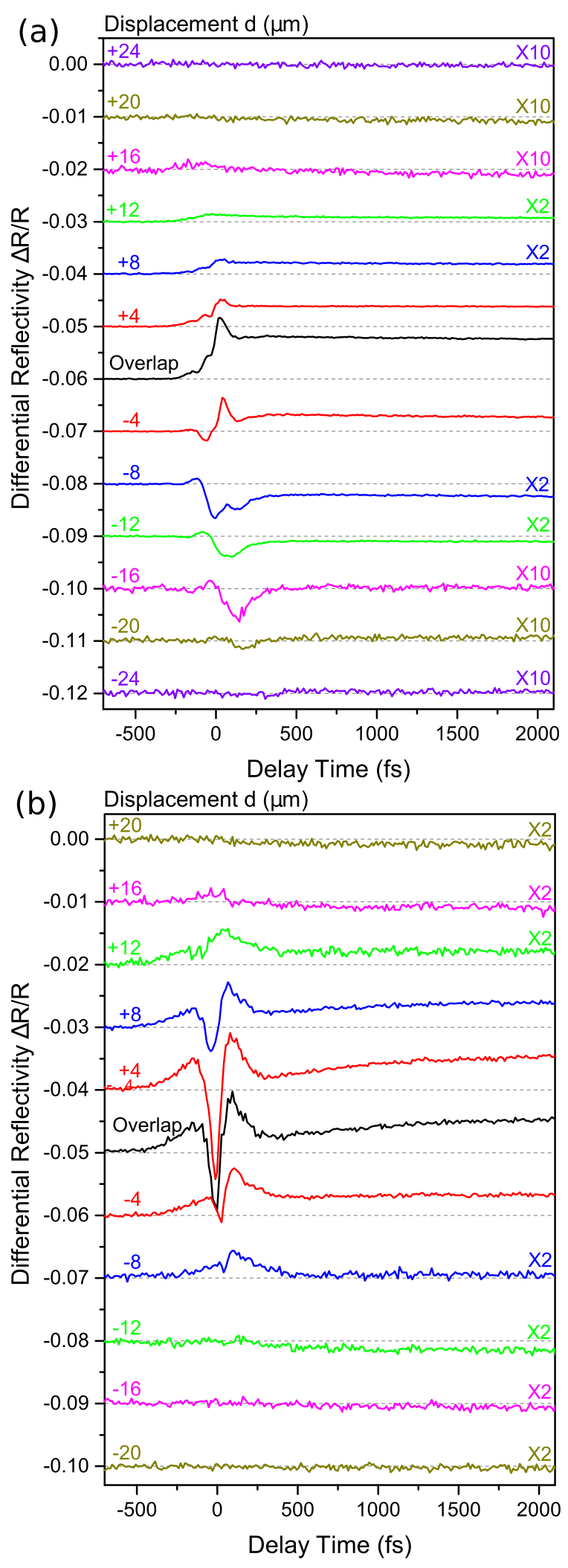

FIG. 5. Transient data for different pump spot positions (scan along the $x$ axis). (a) Grating surface. (b) Bare guiding structure without grating. The labels on the left correspond to the displacement $d$ of the probe spot with respect to the pump spot (in $\mu \mathrm{m})$. The labels on the right side (e.g., $\times 10)$ mark the multiplication factor of each trace to enhance the visibility of the dynamics. For clarity the curves are shifted along the vertical axis. 
The displacement is defined as $\mathbf{d}=\mathbf{r}_{2}-\mathbf{r}_{1}$, where $\mathbf{r}_{1}$ and $\mathbf{r}_{2}$ correspond to the positions of pump and probe spots in the $x y$ plane. It follows that the signal is represented by two distinct contributions. First, there is an instantaneous signal which appears at the temporal coincidence of the pump and probe pulses. This signal resembles a spike, a peak or a dip, centered around zero delay time $\tau=0$. Second, the instantaneous response is followed by a long-lived steplike signal [Fig. 5(a), black curve)] which decays slowly on a much longer timescale of several hundreds of picoseconds, which we attribute to the recombination of excitons. We also see signatures of a decay during several tens of picoseconds which is most likely related to the relaxation of higher-energy carriers.

In case of excitation within the grating coupler, we observe distinct differences between various pump spot positions. Comparing the above described contributions as a function of displacement $d$ in $x$ direction, we conclude that the features behave in different ways. For $d<0$, the steplike response changes the sign at $d \leqslant-8 \mu \mathrm{m}$, whereas for positive $d$ it decreases gradually to zero and disappears in the noise at $d \approx+16 \mu \mathrm{m}$. The instantaneous response which occurs at $\tau=0$ becomes even stronger at $d=-4 \mu \mathrm{m}$ and decays much slower for negative $d$ in comparison to positive displacements. Thus we observe a pronounced asymmetry in the optical response of the exciton resonance with different displacement dependencies for the two contributions. The steplike component is present mainly at positive displacements $(d>0)$ while the instantaneous component is much more pronounced in the opposite direction $(d<0)$.

This asymmetry along the $x$ axis is absent when moving the pump spot along the $y$ axis, i.e parallel to the grating ridges. Here, the shape of the signal measured at $d=0$ in Fig. 5(a) remains the same and only the overall amplitude of the signal decays along both directions in a symmetrical manner. Mapping measurements were also performed on the bare guiding part of the same sample. Figure 5(b) shows such transient signals for different displacements $d$ for the bare structure. Although the amplitude of instantaneous and steplike signals in Fig. 5(b) have different values for positive and negative $d$, the displacement dependencies are symmetric for both signals with a maximum at about $d_{\max }=3 \mu \mathrm{m}$, as will be discussed further.

To quantify the results of the mapping experiments we evaluate the amplitudes $A$ of the instantaneous response at $\tau=0$ and the steplike, long-lived signal. The instantaneous response is an S-shaped feature with a small peak and a subsequent larger peak with opposite sign. The amplitude is taken as the absolute difference between these two peaks' extrema. For some transients, the small peak is absent and only a signal rise before the steplike function appears, here the absolute maximum of this peak is used. For the steplike signal, the magnitude of the contribution is evaluated as the average in the interval from 500 to $1500 \mathrm{fs}$. The corresponding results are shown in Fig. 6 as function of the beam displacement. As mentioned above, we observe an asymmetric behavior of both contributions to the optical response when the scan is performed along the $x$ axis [see Fig. 6(a)] while the asymmetry is absent along the $y$ axis [see Fig. 6(b)] or in either direction on the bare guiding structure [Fig. 6(c)]. Note that all curves are slightly shifted with respect to $d=0$, i.e. the case
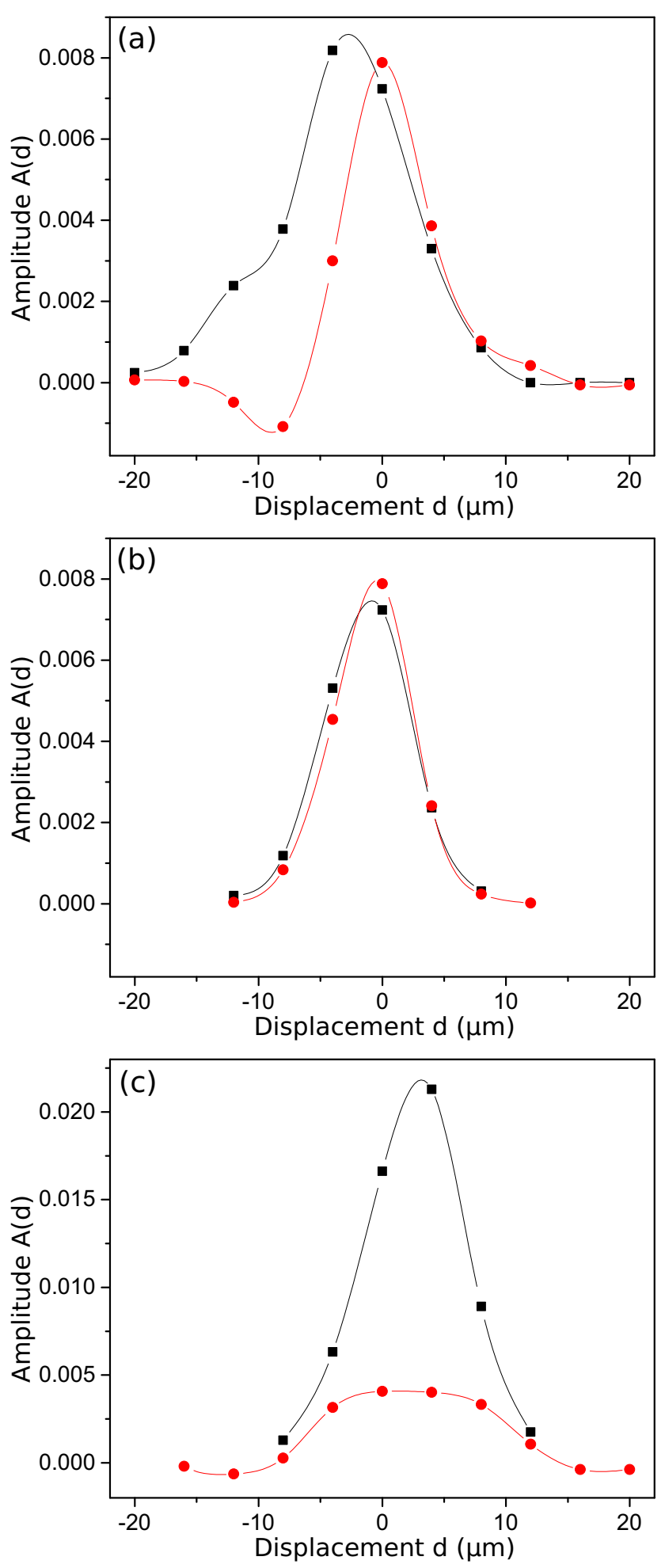

FIG. 6. Amplitude $A$ of the instantaneous signal at zero delay between pump and probe pulses (black squares) and of the steplike signal (averaged in the range $500<\tau<1500 \mathrm{fs}$, red circles) $v s$ the spatial distances between the probe and the pump spots $d$ along the $x$ or $y$ directions. The lines are spline fits given as guides to the eye. (a) shows measurements on the grating with the displacement of the pump along the $x$ axis, (b) is taken on the same position of the grating with the scan along the $y$ axis. In comparison, (c) shows the same measurement as in (a) but on a bare guiding structure area that is not covered with the grating. 
of ideal overlap (about $2-4 \mu \mathrm{m}$ ), which is within the accuracy of the pump beam positioning in our setup. Nevertheless the curves in Figs. 6(b) and 6(c) are symmetric with respect to the position $d_{\max }$ at which the maximum signal is observed. The data indicate that the pump-excited guiding modes propagate along the $x$ axis over distances larger than the spot size. The next section describes the propagation of EP modes in guiding structures and their impact on the optical response in which two main excitation mechanisms are involved.

\section{THEORETICAL DISCUSSION}

In this section, the main experimental findings of Fig. 5(a), namely, the asymmetric optical response of the structure with respect to the pump displacement, will be analyzed and described qualitatively. To highlight the essential nonlinear effects that are responsible for the measured transients, we perform numerical simulations for a rather simple, but transparent model which consists of an one-dimensional chain of two-level systems. Initially we neglect propagation effects and the spatial arrangement of the two-level system accounts for the fixed locations of the displaced pump and probe pulse spots, respectively. Nevertheless, the main properties of the guiding modes as found in the experiment and in the electrodynamic simulation shown in Sec. II are taken into account and enter via their different energies and directions into the calculations. At the end of this section schematic simulations including propagating fields will justify this approach.

In order to understand the essential behavior of the signal $\Delta R / R$, we distinguish three different spatial regions. (1) The transient looks like a step function. It has a maximum near zero delay and then converges towards a positive value for long time delays. This is the case when the system is pumped left from the probe pulse $(d=+4 \mu \mathrm{m}, d=+8 \mu \mathrm{m}, \ldots)$.

(2) The transient shows a sign change for long time delays instead, i.e., a negative signal occurs if the system is pumped right from the probe $(d=-8 \mu \mathrm{m}, d=-12 \mu \mathrm{m}, \ldots)$.

(3) There is an intermediate spatial overlap region showing a quite abrupt change from positive to negative long term signal between $d=-4 \mu \mathrm{m}$ and $d=-8 \mu \mathrm{m}$.

Immediately the question arises why the transients in case 1 and case 2 have different signs even though the system is pumped similarly, however, at opposite sides of the probe. In order to answer this and to distinguish the two situations the actual coupling into the modes that appear due to the gold grating has to be taken into account. In Fig. 2(a), it can be seen that the grating-waveguide system supports two different EP modes. More precisely, for an oblique incidence angle of $\theta=17^{\circ}$ (marked by the vertical dashed line), a mode with central frequency of about $1.63 \mathrm{eV}$ is propagating to the right and another one at about $1.49 \mathrm{eV}$ to the left. The different propagation directions are due to the fact that these modes have opposite slopes in the dispersion relation $\omega(q)$. The off-resonant mode has a negative dispersion, i.e., its direction of propagation is opposite to the wave vector $q$, while the resonant mode has a positive group velocity. The different propagation directions of these modes are one ingredient to explain the spatially asymmetric optical response. In addition, these modes have a very different detuning with respect to

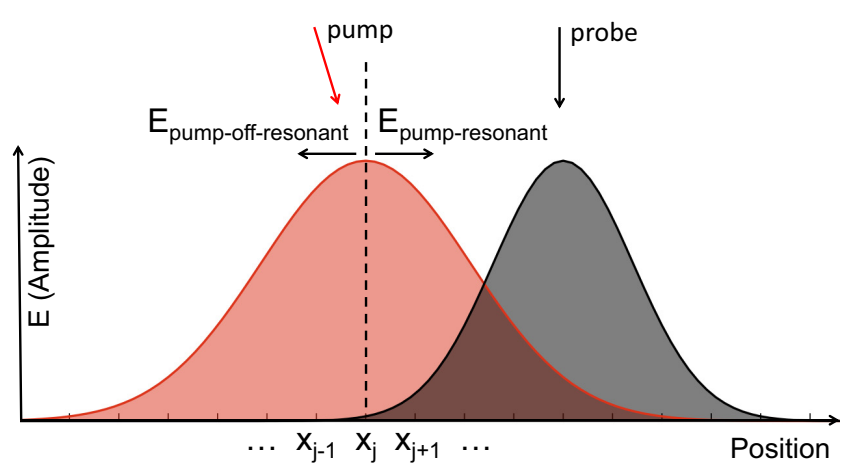

FIG. 7. Configuration of the simulation space. The position of the pump pulse determines where the resonant and the detuned mode, respectively, are present in our model. (For clearer depiction the spot sizes of the pulses are not drawn to scale.)

the exciton at $1.64 \mathrm{eV}$. While the "mode to the right" is basically resonant with the exciton, and thus resonantly generates carriers in the system, the "mode to the left" is detuned by more than $140 \mathrm{meV}$ from the exciton to the low energy side. To model this situation the simulation space is artificially split into two regions within which one part includes the resonant pulse and the other one the detuned pulse, see Fig. 7. As propagation effects are initially neglected the nonlinear interaction in our model takes place only in the overlap region of the pump and the probe pulses.

The simulations of the pump-probe experiment are performed in the lowest-order $\chi^{(3)}$ limit, i.e., the probe is taken into account linearly and the pump in second order [24-26]. To model the experimental findings we use the following Bloch equations which describe the position-dependent excitations of the collection of two-level systems:

$$
\begin{aligned}
\frac{\partial}{\partial t} p_{j}^{(1)}(t) & =\left(-i \omega_{R}-\gamma_{p}\right) p_{j}^{(1)}(t)+i \frac{\mu}{\hbar} E\left(x_{j}, t\right), \\
\frac{\partial}{\partial t} n_{j}^{(2)}(t)= & -2 \frac{\mu}{\hbar} \operatorname{Im}\left(E\left(x_{j}, t\right) p_{j}^{*(1)}(t)\right)-\gamma_{n} n_{j}^{(2)}(t), \\
\frac{\partial}{\partial t} p_{j}^{(3)}(t)= & \left(-i \omega_{R}-\gamma_{p}\right) p_{j}^{(3)}(t)-2 i \frac{\mu}{\hbar} n_{j}^{(2)} E\left(x_{j}, t\right) \\
& -\alpha n_{j, \text { two-photon }}(t) p_{j}^{(1)}(t) .
\end{aligned}
$$

Here, with index $j$ denoting the position, $p^{(1)}$ is the linear microscopic polarization, $n^{(2)}$ the density created via onephoton absorption, and $p^{(3)}$ is the nonlinear polarization determining the differential reflectivity signal. As shown in Ref. [12], for detuned pumping two-photon excitations may dominate the nonlinear propagation and therefore we include $n_{\text {two-photon }}$ which is generated through two-photon absorption into our model. Further quantities are the resonance energy of the two-level system $\hbar \omega_{R}=\hbar \omega_{\text {exciton }}=1.64 \mathrm{eV}$, the phenomenological dephasing constant $\gamma_{p}=2 /(1 \mathrm{ps})$, the relaxation rate $\gamma_{n}=2 /(10 \mathrm{ps})$, the dipole-matrix element $\mu$, the phenomenological constant $\alpha$ representing the strength of the so-called excitation-induced dephasing process [25,27-29] which here is caused by two-photon excitations [12]. Whereas the absolute values of $\mu$ and $\alpha$ are not relevant, their ratio, or more precisely the ratio between the different nonlinearities 
arising from one- and two-photon excitation is adjusted in order to describe the experimental results. $E(t)$ refers to either the pump or probe field, respectively. The set of differential equations (1)-(3) is solved and the sum $\mathcal{P}(t)=\sum_{l} \mu p_{l}^{(3)}(t)$ over each grid point $l$ is computed. After performing a Fourier transform and applying a spectral filter $f$, the quantity $[-\operatorname{Im}(\mathcal{P}(\omega) f(\omega))]$ is proportional to the differential reflectivity signal $\Delta R / R(\omega)$ for short probe pulses [30,31]. Details of the numerical procedure are given in Appendix.

First, case 1 ("pump left from the probe") is examined. To understand the long time behavior of the transients it is important to note that the inhomogeneities in Eqs. (1) and (3) have different signs. Whereas $p^{(1)}(t)$ is driven by $+i E(t)$, meaning that a field generates a first-order polarization and subsequently a density of real carriers $n^{(2)}(t)$ in the system, the third-order polarization is mainly determined by $-i n^{(2)} E(t)$, since the term $\alpha n_{\text {two-photon }} p^{(1)}$ is negligible for resonant excitation, see below. The different signs mean that, if carriers already exist in the material, the overall absorption will decrease. This is pump-induced bleaching which arises due to phase space filling (Pauli blocking) by the pump-induced carriers $[23,25,26]$. Bleaching will lead to a negative sign in the differential absorption and thus to a positive transient in the $\Delta R / R$ signal. The simulation results in Fig. 8 for $d=+4 \mu \mathrm{m},+8 \mu \mathrm{m}$, etc. show the corresponding behavior. Note that there is a slight shift in the pump position between theory and experiment which is due to the uncertainty in the zero point of the displacement axis in the experimental setup. The color code of the individual curves indicates that the difference is about $4 \mu \mathrm{m}$, i.e., similar to the spot size of the laser pulses.

In case 2 ("pump right from the probe") the overlap between the probe pulse and the detuned pump pulse plays a decisive role. It is known for this particular material system that for far off-resonant excitation the so called excitationinduced dephasing [27] (EID) instead of Pauli blocking (PB) is the dominant nonlinearity. It has been suggested that for off-resonant excitation conditions EID is mainly caused by carriers that are generated via two-photon absorption [12]. For simplicity we model the density generated by two-photon absorption by an additional set of differential equations of type (1) and (2) which are resonantly tuned to the pump with a squared amplitude. As for real two-photon absorption the generated density $n_{\text {two-photon }}$ rises with the integral over the pump pulse which is the most relevant feature for the following analysis [32]. Examining Eq. (3), it is clear, however, that the inhomogeneity containing $n_{\text {two-photon }}$ can only contribute in the off-resonant case significantly since in this case the density of real carriers $n^{(2)}$ is small. To illustrate the difference between PB and EID, Fig. 9 shows the spectral behavior of $\operatorname{Im}\left(p^{(3)}(\omega)\right)$ for a single two-level system when it is either driven by $-i n^{(2)} E(t)$ or $-\alpha n^{(2)} p^{(1)}$. Instead of pure bleaching for $\mathrm{PB}$, which has a negative Lorentzian line shape, with EID the spectrum broadens and thus develops also positive contributions above and below the resonance. As in the experiments the computed spectra are filtered below the resonance at $760 \mathrm{~nm}$ and therefore it is possible to reproduce the opposite signs of the two cases 1 and 2 by including PB and EID. EID is also responsible for the negative long term signal in the transients.

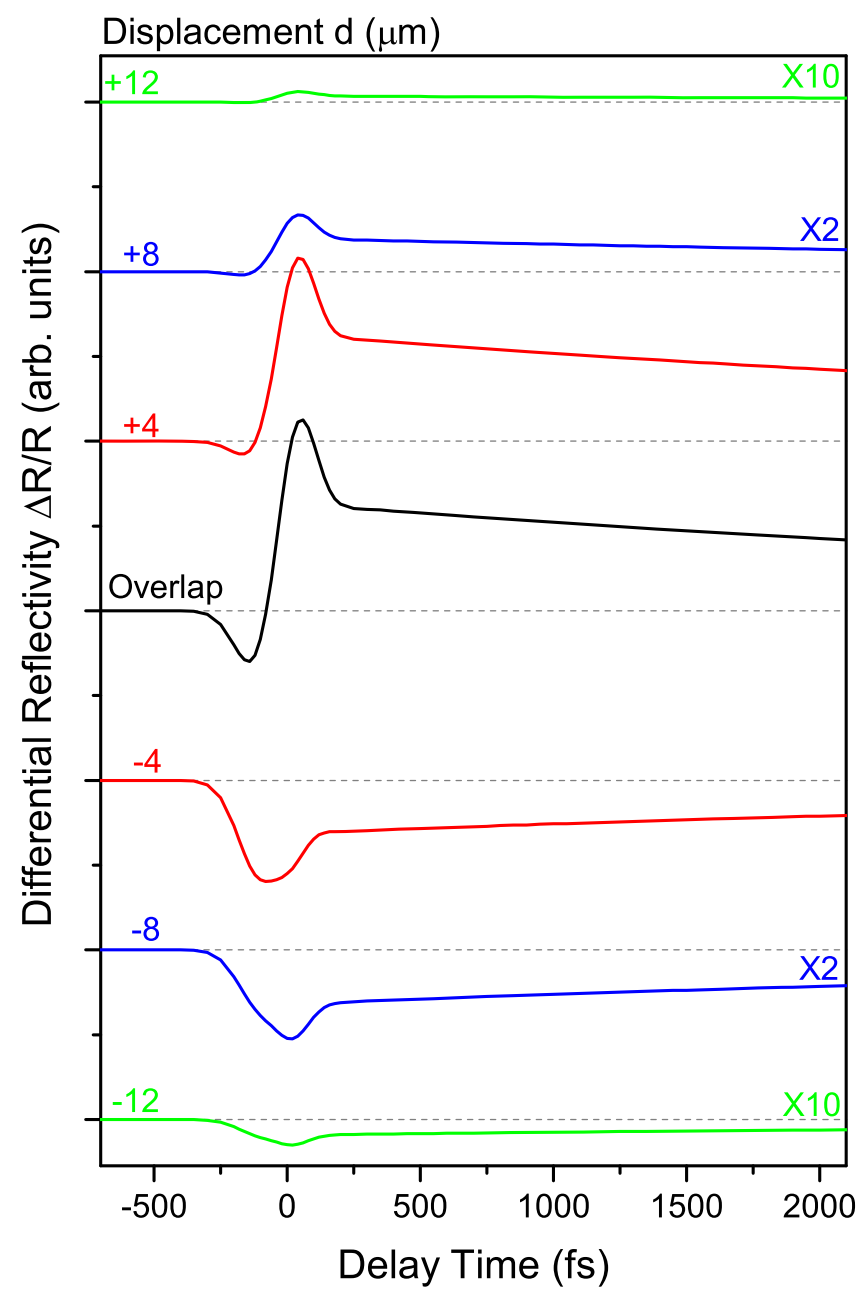

FIG. 8. Simulation results for a one-dimensional arrangement of two-level systems in the $\chi^{(3)}$ limit showing the $\Delta R / R$ transient analogously to the measurement in Fig. 5(a). The model reproduces the different experimental situations, i.e., the cases 1, 2, and 3 as described in the text.

The abrupt sign change in case 3 of the $d=-4$ and $-8 \mu \mathrm{m}$ curves in Fig. 5(a) is due to the fact that the pump pulse separates the detuned and the resonant mode. Because the spot radii are about $4-5 \mu \mathrm{m}$, a change between the two regimes on this length scale is plausible. For the overall comparison in Fig. 10, the amplitude dependence on the spatial distances is plotted for the instantaneous signal and the long time behavior analogously to the experimental curves in Fig. 6(a). The general structure of the measurements is reproduced quite well by the one-dimensional model. Merely the widths of the curves in Fig. 10 appear to be slightly too narrow and the positions somewhat shifted.

Finally, we demonstrate that the features obtained from the model described above are still preserved also with propagation. Full three-dimensional numerical solutions of the Maxwell-Bloch equations are generally very demanding. Therefore here schematic one-dimensional propagation calculations are performed to underpin that the PB and EID contributions survive when the pump truly moves through the region of probe's spot. In the performed Maxwell-Bloch 


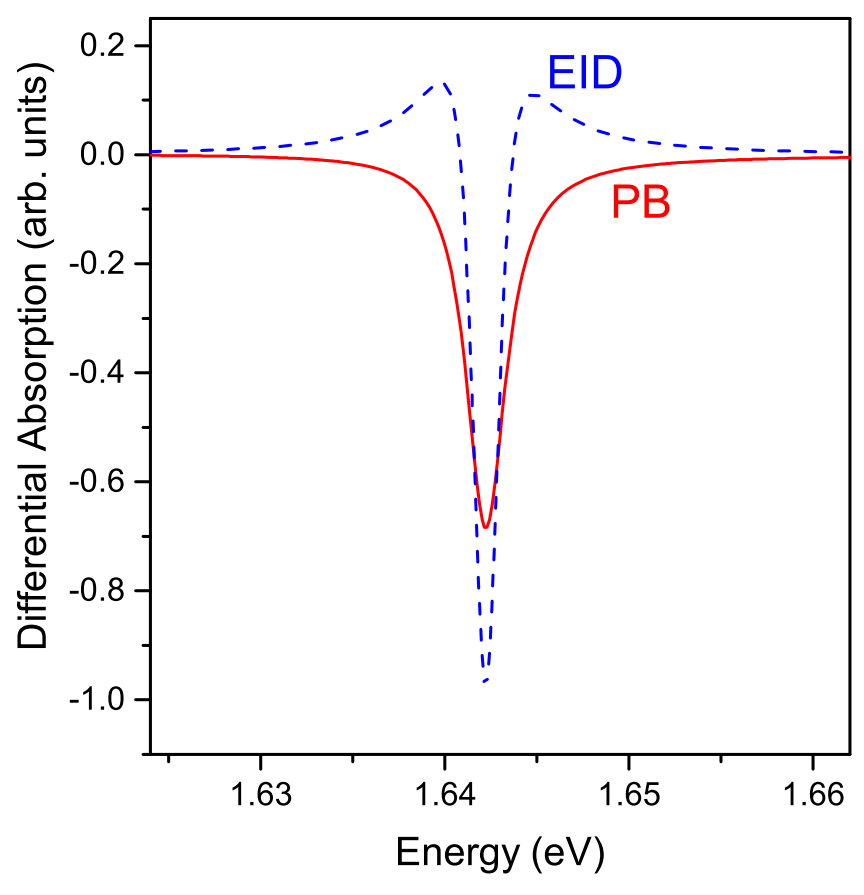

FIG. 9. Schematic comparison of the differential absorption $\operatorname{Im}\left[p^{(3)}(\omega)\right]$ when generated by Pauli blocking (red solid line) or by excitation-induced dephasing (blue dashed line). The sign slightly below the resonance is responsible for the long-term behavior of the transient because this part of the spectrum passes through the filter used in the experiments.

simulations, the pump and probe pulses are spatially well separated $(\geqslant 16 \mu \mathrm{m})$. Then, for the pump pulse the one-dimensional Maxwell-Bloch equations are solved with the FDTD scheme [33] using the method described in Refs. [34,35] whereas the probe pulse is considered to stay at a fixed position. Thus, unlike in the above simplified analysis where the center of the pump and probe pulses were station-

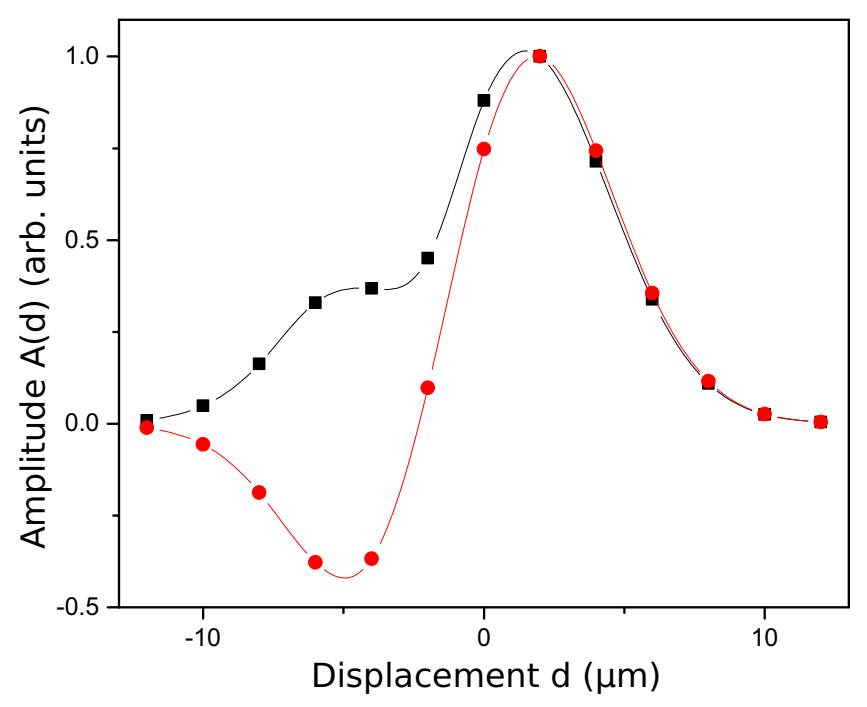

FIG. 10. Amplitudes of the instantaneous signal (black squares) and the long term behavior (red circles) analogously to the experiment of Fig. 6(a).
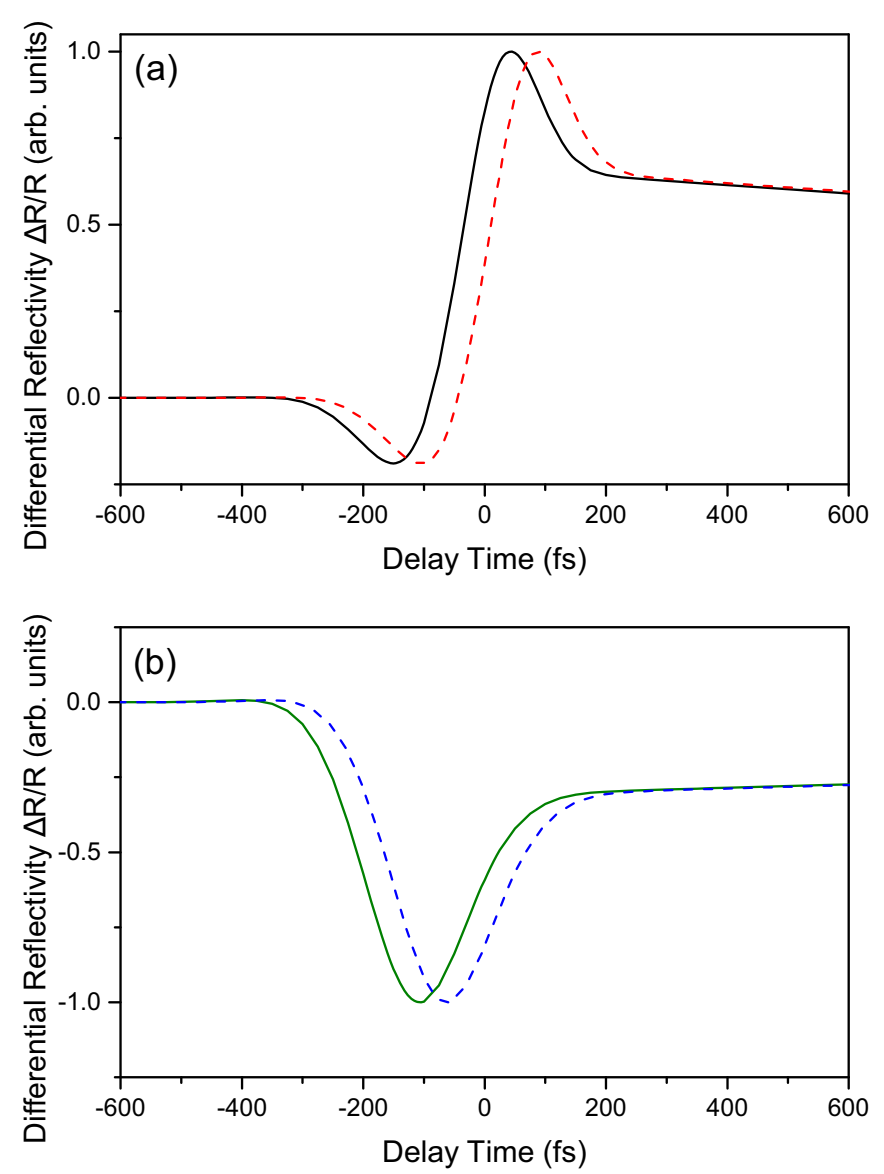

FIG. 11. Results of one-dimensional FDTD-simulations for twolevel systems in the $\chi^{(3)}$ limit showing the $\Delta R / R$ transients for (a) larger positive displacements (black solid line $d=+16 \mu \mathrm{m}$, red dashed line $d=+20 \mu \mathrm{m}$ ). The system is pumped at the left (case 1). (b) shows larger negative distances (green solid line $d=-16 \mu \mathrm{m}$, blue dashed line $d=-20 \mu \mathrm{m}$ ). Here, the system is pumped at the right (case 2).

ary, the pump pulse propagates from left to right or reversely and passes the region of the probe. If the propagation direction is correctly associated with the resonant or off-resonant frequency, respectively, the results in Figs. 11(a) and 11(b) are obtained. They still show positive or negative long time signals, respectively, like those obtained in the model without propagation. Therefore, one can conclude that propagation effects change the signals only slightly and that the main features of the mere $\chi^{(3)}$ model, i.e., Eqs. (1)-(3), are well reproduced also with propagation. It should be pointed out that the difference in the simulations "with" and "without" propagation is the spatial spreading of the pump pulse. Of course, both models rely on polariton effects as the off/resonant excitation plays a major role. Whereas the first approach manually distributes the pump profile the FDTD calculation self-consistently propagates the field. A relatively weak dispersion results in only a slight impact on the transients.

It should be noted that we did not introduce any losses in our one-dimensional propagation calculations. While this could be done, we refrained from including losses since we basically wanted to demonstrate that propagation does not 
qualitatively modify the above described findings and thus that they should in principle be observable for longer displacements, if losses are not too large.

\section{SUMMARY}

In conclusion, we have demonstrated an efficient coupling of 30-fs pulses with large bandwidth into a guiding CdZnTe semiconductor layer using a metallic grating. For the case of TM modes, the guiding modes are hybridized with plasmonic excitations in the metallic grating. This leads to a significant increase of the coupling efficiency for $p$-polarized incident light. Compared to TE modes the width and strength of the guiding resonances observed in the reflectivity spectra are increased several times leading to resonant reflectivity features with $20-30 \mathrm{~nm}$ spectral bandwidth. This is especially important for fast operation at subpicosecond timescales in photonic structures where short pulses with large bandwidth should be transmitted. In addition, using angle-resolved reflection spectroscopy we were able to map the dispersion of both TM and TE modes. Here, the strong bending of the dispersion curves at photon energies close to the exciton resonance in the CdZnTe guiding layer indicates the typical EP behavior with a strong decrease in the group velocity.

Guiding structures covered with Bragg metallic gratings make it possible to excite propagating exciton-polaritons in the direction perpendicular to the grating slits. If the excitation laser spot is smaller than the propagation length of the exitonpolariton mode the influence on the optical properties with respect to the position of the laser excitation is asymmetric. The asymmetry occurs only when the pump beam has oblique incidence. In this case due to momentum conservation of the in-plane wave vector only exciton-polaritons which propagate in one direction will be excited. Thereby the grating makes the momentum conservation possible and excitation of the guiding exciton-polariton modes takes place. In case if several modes are present, they may have different propagation directions with respect to the incidence beam which is dictated by the sign of their group velocity. Thereby the impact on the optical properties by each of these modes may be different. This is what happens in our particular case where two modes (on-resonant and off-resonant) have opposite group velocities and each of them is responsible for a different excitation process which takes place in the guiding layer during their propagation. Note that under zero incidence angle a standing wave is excited and the excitation induced asymmetry disappears.

Using time-resolved pump-probe differential reflectivity measurements with a spatial resolution of several micrometers we have studied the influence of off-resonant and on-resonant EP propagation on the exciton absorption spectrum in the guiding layer. We demonstrate that there are two optical regimes which depend on the direction of propagation of the pump-induced EPs inside the guiding layer (resonant and off-resonant modes). The impact on the exciton absorption is opposite for each of the regimes leading to significant asymmetries in the spatially resolved transients. Theoretical modeling using two-level systems interacting with the light field in the lowest order approximation allows us to qualitatively reproduce the experimental results by taking two nonlinear processes into account, namely Pauli blocking and excitationinduced dephasing, which dominate in the on-resonant and off-resonant cases, respectively. In case of resonant excitation Pauli blocking leads to bleaching of the absorption and thus the reflection (transmission) is increased. For off-resonant polaritons excitation-induced dephasing leads to a broadening of the exciton resonance and for detuned detection the absorption increases leading to an attenuation of the transmitted or reflected probe pulse. The combination of these two contributions can result in a pronounced sub-ps instantaneous response at the temporal pump-probe overlap which manifests itself in abrupt changes of the differential reflection showing a change of the sign. The further temporal response is governed by the population dynamics of the photoexcited carriers on a time scale of ten to hundred picoseconds. We stress that the spatially asymmetric optical response is a result of the guiding structures where the in-plane propagation can be tailored by the grating parameters and the spectral shape of the incident pulse. Such features can potentially be used for the realization of multiplexing with different colors of control pulses.

We note that while semiconductor planar guiding structures posses strong nonlinearities in the spectral range close to the exciton resonance they require low temperatures. Moreover the refractive index contrast between the CdZnTe and $\mathrm{CdMgTe}$ layers in II-VI semiconductor compounds has to be increased in order to improve the light-matter coupling. This point can be resolved by growth of II-VI semiconductor guiding layers directly on lattice matched $\mathrm{BaF}_{2}$ substrates [36].

\section{ACKNOWLEDGMENTS}

We thank W. Iqbal for assistance during the measurements. We acknowledge financial support from the Deutsche Forschungsgemeinschaft (DFG) through the Collaborative Research Centre TRR 142 (Project No. 231447078, Project No. A02).

\section{APPENDIX: NUMERICAL PARAMETERS}

The dynamics of the macroscopic polarization $\mathcal{P}(t)$ is determined via the set of coupled differential Eqs. (1)-(3).

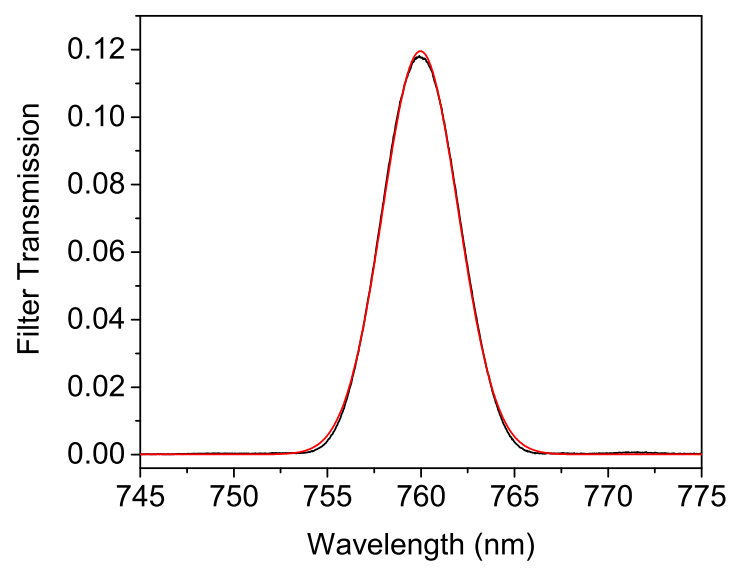

FIG. 12. Measured linear transmission spectrum through the filter (black line) and corresponding Gaussian fit (red line). 
As all involved optical pulses have finite duration and the material excitations decay with $\gamma_{p, n}$ it is sufficient to numerically integrate them in a limited time window. Here, we use a start time $t_{s}=-10 \mathrm{ps}$ and an end time $t_{e}=40 \mathrm{ps}$ where $t=0$ corresponds to the time of arrival of the probe pulse.

$$
P_{\tau}(\omega)=\int_{-\infty}^{\infty} P_{\tau}(t) e^{i \omega t} d t \rightarrow \int_{t_{s}}^{t_{e}} P_{\tau}(t) e^{i \omega t} d t
$$

In order to obtain the transient $T_{\tau}$ for a certain delay $\tau$, we also take the properties of the optical filter used in the experiment into account. Figure 12 shows that a Gaussian fit to the linear transmission spectrum is well suited to represent the filter function $f(\omega)$. The maximum of the transmission is slightly below the exciton resonance at around $760 \mathrm{~nm}$ with a width of about $4.7 \mathrm{~nm}$. Numerically we integrate far above/below the maximum from a lower limit $\omega_{l}$ corresponding to $880 \mathrm{~nm}$ to an upper limit $\omega_{u}$ corresponding to $650 \mathrm{~nm}$ yielding the transient

$$
T_{\tau}=-\operatorname{Im}\left(\int_{\omega_{l}}^{\omega_{u}} P_{\tau}(\omega) f(\omega) d \omega\right)
$$

[1] O. Benson, Assembly of hybrid photonic architectures from nanophotonic constituents, Nature (London) 480, 193 (2011).

[2] P. Cheben, R. Halir, J. H. Schmid, H. A. Atwater, and D. R. Smith, Subwavelength integrated photonics, Nature (London) 560, 565 (2018).

[3] J. Leuthold, C. Koos and W. Freude, Nonlinear silicon photonics, Nat. Photon. 4, 535 (2010).

[4] D. Sanvitto and S. Kéna-Cohen, The road towards polaritonic devices, Nat. Mater. 15, 1061 (2016).

[5] S. T. Cundiff, Coherent spectroscopy of semiconductors, Opt. Express 16, 4639 (2008).

[6] S. I. Pekar, The theory of electromagnetic waves in a crystal in which excitons are produced, Zh. Eksp. Teor. Fiz. 33, 1022 (1957) [Sov. Phys. JETP 6, 785 (1958)].

[7] J. J. Hopfield, Theory of the contribution of excitons to the complex dielectric constant of crystals, Phys. Rev. 112, 1555 (1958).

[8] T. V. Shubina, M. M. Glazov, A. A. Toropov, N. A. Gippius, A. Vasson, J. Leymarie, A. Kavokin, A. Usui, J. P. Bergman, G. Pozina, and B. Monemar, Resonant Light Delay in GaN with Ballistic and Diffusive Propagation, Phys. Rev. Lett. 100, 087402 (2008).

[9] T. V. Shubina, M. M. Glazov, N. A. Gippius, A. A. Toropov, D. Lagarde, P. Disseix, J. Leymarie, B. Gil, G. Pozina, J. P. Bergman, and B. Monemar, Delay and distortion of slow light pulses by excitons in ZnO, Phys. Rev. B 84, 075202 (2011).

[10] T. Godde, I. A. Akimov, D. R. Yakovlev, H. Mariette, and M. Bayer, Subnanosecond delay of light in CdZnTe crystals, Phys. Rev. B 82, 115332 (2010).

[11] T. Godde, M. M. Glazov, I. A. Akimov, D. R. Yakovlev, H. Mariette, and M. Bayer, Magnetic field induced nutation of exciton-polariton polarization in (Cd, Zn)Te crystals, Phys. Rev. B 88, 155203 (2013).

[12] J. Lohrenz, S. Melzer, C. Ruppert, I. A. Akimov, H. Mariette, M. Reichelt, A. Trautmann, T. Meier and M. Betz, Ultrafast dynamical response of the lower exciton-polariton branch in CdZnTe, Phys. Rev. B 93, 075201 (2016).

[13] M. Lopez-Garcia, Y.-L. D. Ho, M. P. C. Taverne, L.-F. Chen, M. M. Murshidy, A. P. Edwards, M. Y. Serry, A. M. Adawi, J. G. Rarity, and R. Oulton, Efficient out-coupling and beaming of Tamm optical states via surface plasmon polariton excitation, Appl. Phys. Lett. 104, 231116 (2014).

[14] Z. Wang, B. Zhang, and H. Deng, Dispersion Engineering for Vertical Microcavities Using Subwavelength Gratings, Phys. Rev. Lett. 114, 073601 (2015).
[15] P. M. Walker, L. Tinkler, M. Durska, D. M. Whittaker, I. J. Luxmoore, B. Royall, D. N. Krizhanovskii, M. S. Skolnick, I. Farrer, and D. A. Ritchie, Exciton polaritons in semiconductor waveguides, Appl. Phys. Lett. 102, 012109 (2013).

[16] P. Yu. Shapochkin, M. S. Lozhkin, I. A. Solovev, O. A. Lozhkina, Y. P. Efimov, S. A. Eliseev, V. A. Lovcjus, G. G. Kozlov, A. A. Pervishko, D. N. Krizhanovskii, P. M. Walker, I. A. Shelykh, M. S. Skolnick, and Yu. V. Kapitonov, Polarization-resolved strong light-matter coupling in planar GaAs/AlGaAs waveguides, Opt. Lett. 43, 4526 (2018).

[17] P. M. Walker, L. Tinkler, D. V. Skryabin, A. Yulin, B. Royall, I. Farrer, D. A. Ritchie, M. S. Skolnick, and D. N. Krizhanovskii, Ultra-low-power hybrid light-matter solitons, Nat. Commun. 6, 8317 (2015).

[18] A. Christ, S. G. Tikhodeev, N. A. Gippius, J. Kuhl, and H. Giessen, Waveguide-Plasmon Polaritons: Strong Coupling of Photonic and Electronic Resonances in a Metallic Photonic Crystal Slab, Phys. Rev. Lett. 91, 183901 (2003).

[19] CST STUDIO SUITE 2015, Release Version 2015.06

[20] R. André and Le Si Dang, Low-temperature refractive indices of $\mathrm{Cd}_{1-x} \mathrm{Mn}_{x} \mathrm{Te}$ and $\mathrm{Cd}_{1-y} \mathrm{Mg}_{y}$ Te, J. Appl. Phys. 82, 5086 (1997).

[21] B. Xu, J. M. Gunn, J. M. D. Cruz, V. V. Lozovoy, and M. Dantus, Quantitative investigation of the multiphoton intrapulse interference phase scan method for simultaneous phase measurement and compensation of femtosecond laser pulses, J. Opt. Soc. Am. B 23, 750 (2006).

[22] L. E. Kreilkamp, I. A. Akimov, V. I. Belotelov, B. A. Glavin, L. V. Litvin, A. Rudzinski, M. Kahl, R. Jede, M. Wiater, T. Wojtowicz, G. Karczewski, D. R. Yakovlev, and M. Bayer, Terahertz dynamics of lattice vibrations in Au/CdTe plasmonic crystals: Photoinduced segregation of Te and enhancement of optical response, Phys. Rev. B 93, 125404 (2016).

[23] M. Joffre, D. Hulin, A. Migus, A. Antonetti, C. Benoit à la Guillaume, N. Peyghambarian, M. Lindberg, and S. W. Koch, Coherent effects in pump-probe spectroscopy of excitons, Opt. Lett. 13, 276 (1988).

[24] M. Lindberg and S. W. Koch, Theory of coherent transients in semiconductor pump-probe spectroscopy, J. Opt. Soc. Am. B 5, 139 (1988).

[25] H. Haug and S. W. Koch, Quantum Theory of the Optical and Electronic Properties of Semiconductors, 5th ed. (World Scientific, Singapore, 2009).

[26] T. Meier, P. Thomas, and S. W. Koch, Coherent Semiconductor Optics: From Basic Concepts to Nanostructure Applications (Springer, New York, 2007). 
[27] H. Wang, K. Ferrio, D. G. Steel, Y. Z. Hu, R. Binder, and S. W. Koch, Transient Nonlinear Optical Response from Excitation Induced Dephasing in GaAs, Phys. Rev. Lett. 71, 1261 (1993).

[28] Y. Z. Hu, R. Binder, S. W. Koch, S. T. Cundiff, H. Wang, and D. G. Steel, Excitation and polarization effects in semiconductor four-wave-mixing spectroscopy, Phys. Rev. B 49, 14382 (1994).

[29] L. Bányai, Q. T. Vu, and H. Haug, Excitation induced dephasing in four-wave mixing and Coulomb quantum kinetics, Phys. Rev. B 58, R13341(R) (1998).

[30] I. Balslev, R. Zimmermann, and A. Stahl, Two-band densitymatrix approach to nonlinear optics of excitons, Phys. Rev. B 40, 4095 (1989).

[31] K. Leo, J. Shah, T. C. Damen, A. Schulze, T. Meier, S. SchmittRink, P. Thomas, E. O. Göbel, S. L. Chuang, M. S. C. Luo, W. Schäfer, K. Köhler, and P. Ganser, Dissipative dynamics of an electronic wavepacket in a semiconductor double well potential, IEEE J. Quantum Electronics 28, 2498 (1992).
[32] The fact that $n_{\text {two-photon }}$ is quadratic in the pump field whereas a real two-photon occupation is of fourth-order does not play a significant role in our discussion, since the different strengths of the excitations are adjusted by choosing the parameter $\alpha$ appropriately.

[33] A. Taflove and S. C. Hagness, Computational Electrodynamics The Finite-Difference Time-Domain Method, 3rd ed. (Artech House, Norwood, 2005).

[34] R. W. Ziolkowski, The incorporation of microscopic material models into the FDTD approach for ultrafast optical pulse simulations, IEEE Trans. Antennas Propag. 45, 375 (1997).

[35] R. W. Ziolkowski, J. M. Arnold, and D. M. Gogny, Ultrafast pulse interactions with two-level atoms, Phys. Rev. A 52, 3082 (1995).

[36] E. Janik, E. Dynowska, J. Bąk-Misiuk, J. Domagała, M. Kutrowski, T. Wojtowicz, and A. Stachow, Zinc-blende $\mathrm{MnTe}(111)$ on $\mathrm{BaF}_{2}$ (111) substrates for optical measurements, Appl. Phys. Lett. 68, 3796 (1996). 